\title{
FAST REGISTRATION OF TERRESTRIAL LIDAR POINT CLOUD AND SEQUENCE IMAGES
}

\author{
Jie Shao ${ }^{\text {a }}$, Wuming Zhang ${ }^{\text {a }}$, Yaqiao Zhu ${ }^{\text {b }}$, Aojie Shen ${ }^{\text {a }}$ \\ ${ }^{a}$ State Key Laboratory of Remote Sensing Science, Institute of Remote Sensing Science and Engineering, Faculty of Geographical \\ Science, Beijing Normal University, Beijing 100875, China - shaojie@ mail.bnu.edu.cn \\ ${ }^{\mathrm{b}}$ College of Urban and Environment, Hubei Normal University, Huangshi 435002, Hubei, China - yaqiaozhu@163.com
}

\section{Commission III, WG III/6}

KEY WORDS: Terrestrial LiDAR, Point cloud, Sequence Images, 2D-3D, Registration

\begin{abstract}
:
Image has rich color information, and it can help to promote recognition and classification of point cloud. The registration is an important step in the application of image and point cloud. In order to give the rich texture and color information for LiDAR point cloud, the paper researched a fast registration method of point cloud and sequence images based on the ground-based LiDAR system. First, calculating transformation matrix of one of sequence images based on 2D image and LiDAR point cloud; second, using the relationships of position and attitude information among multi-angle sequence images to calculate all transformation matrixes in the horizontal direction; last, completing the registration of point cloud and sequence images based on the collinear condition of image point, projective center and LiDAR point. The experimental results show that the method is simple and fast, and the stitching error between adjacent images is litter; meanwhile, the overall registration accuracy is high, and the method can be used in engineering application.
\end{abstract}

\section{INTRODUCTION}

At present, most LiDAR systems usually consist of a laser scanner and camera. Laser scanner is used for acquisition of three-dimensional (3D) spatial geometric and intensity information; camera is used for acquisition of sequence color images by revolving around a fixed axis. Registration of LiDAR point cloud and two-dimensional (2D) color image can enhance visualization and identifiability of $3 \mathrm{D}$ point cloud, and help object recognition and extraction (Alex et al, 2013). Sequence images are composed by a set of images, but seamless registration of LiDAR point cloud and sequence images is difficult to solve.

To solve the problem of 2D-3D registration, a lot of methods have been developed (Mishra and Zhang, 2012). Due to a stereo pair of optical images can be used for 3D reconstruction by using photogrammetry techniques (Liu et al, 2006) and stereo vision (Sirmacek et al, 2013), the problem of image-to-point cloud registration can be changed into 3D-3D registration (Zhao et al, 2005). In this research direction, SIFT algorithm (Lowe, 2004; B“"ohm and Becker, 2007) is usually used for correspondent point extraction; and then 3D reconstruction is applied based on correspondent point pairs; last, ICP (Chen and Medioni, 1991; Besl and Mckay, 1992) is used for the registration of $3 \mathrm{D}$ dense point cloud from a pair of adjacent images and 3D LiDAR point cloud ( $\mathrm{Li}$ and Low, 2009). However, these methods are complicated, and accuracy of $3 \mathrm{D}$ reconstruction is easy affected by wrong correspondent point pairs. Several researches focused on the calculation of the 
transformation matrix between $2 \mathrm{D}$ optical image and 3D LiDAR point cloud (Shao et al, 2013). The transformation matrix is decided by elements of exterior orientation which consist of position and attitude information. In general, GPS/INS is used for the acquisition of image position and attitude information (Swart et al, 2011), but there are several drawbacks: GPS reception is poor in environments where includes many trees area and urban canyons, and these drawbacks are not useful for field measurement; besides, GPS and IMU are expensive, and increase the cost.

The essence of image-to-point cloud registration is to decide transformation relation between $2 \mathrm{D}$ and $3 \mathrm{D}$ in the terrestrial LiDAR system. However, transformation matrixes between sequence images and LiDAR point cloud are difficult to calculate, and most of the calculation methods are complicated. In order to simplify the computational process, the paper proposed a simple and fast algorithm for calculation of transformation matrixes based on the inherent geometric relations among sequence images.

\section{METHODS}

The goal of the paper is to complete registration of terrestrial LiDAR point cloud and sequence images. In fact, the essence is to calculate all rigid transformation matrixes between $3 \mathrm{D}$ LiDAR point cloud of and 2D optical image. First, the paper calculates an accurate transformation matrix between one of the sequence images and point cloud based on collinearity relationship of laser point to complete 2D-3D registration, image point and projective center; then, the paper calculates other transformation matrixes based on the fixed location relationship between sequence images.

\subsection{D-3D registration}

The collinearity relationship between 2D pixel coordinates of optical image and 3D point cloud coordinates of LiDAR data is physically meaningful and rigorous (Zhang et al, 2015), and is usually expressed in the form of transformation matrix which consists of translation vector and rotation matrix. The translation vector of $\mathrm{x}, \mathrm{y}, \mathrm{z}$ coordinates represents the location relations of coordinate systems between LiDAR and camera; the rotation matrix which is calculated by the angle of roll, pitch and yaw, represents the pose of $2 \mathrm{D}$ optical image in
LiDAR coordinate system. The collinearity equation between 2D pixel coordinates and $3 \mathrm{D}$ point cloud coordinates is expressed by Eq. (1).

$$
X_{\text {pixel }}=R_{\text {camera }} R_{T} X_{\text {LiDAR }}
$$

Where $\boldsymbol{X}_{\text {pixel }}$ : pixel coordinates on optical image $\left(X_{\text {pixel }}=\left[\begin{array}{ll}u & v\end{array}\right]^{T}\right)$

$\boldsymbol{R}_{\text {camera }}$ : camera intrinsic matrix

$\boldsymbol{R}_{\boldsymbol{T}}$ : transformation matrix between $2 \mathrm{D}$ pixel coordinates and $3 \mathrm{D}$ point cloud coordinates of, $R_{T}=\left[\begin{array}{ll}R & T \\ 0 & 1\end{array}\right]$ ( $\boldsymbol{R}$ is rotation matrix, and expressed using Rodrigues matrix; $\boldsymbol{T}$ is translation vector)

$\boldsymbol{X}_{\text {LiDAR }}$ : 3D point cloud coordinates of LiDAR $\left(X_{\text {LiDAR }}=\left[\begin{array}{lll}X & Y & Z\end{array}\right]^{T}\right)$

The Eq. (1) can be expanded using Eq. (2)

$$
\left[\begin{array}{l}
u \\
v \\
1
\end{array}\right]=\left[\begin{array}{ccc}
f_{x} & 0 & u_{0} \\
0 & f_{y} & v_{0} \\
0 & 0 & 1
\end{array}\right]\left[\begin{array}{cc}
R & T \\
0 & 1
\end{array}\right]\left[\begin{array}{c}
X \\
Y \\
Z \\
1
\end{array}\right]
$$

Where $u_{0}, v_{0}$ : image coordinates of camera's principal point

$f_{x}, f_{y}$ : focal length of the horizontal and vertical axis on optical image.

In Eq. (2), the camera intrinsic parameters can be got using camera calibration. $\boldsymbol{X}_{\text {pixel }}$ and $\boldsymbol{X}_{\boldsymbol{L I D A R}}$ are the coordinate values of correspondent feature point, and can be set as known parameters. If there are at least three pairs of correspondent feature points, we can calculate the unknown rotation matrix and translation vector.

\subsection{Registration of LiDAR point cloud and sequence images}

The essence of registration between LiDAR point cloud and sequence optical images is to calculate all transformation matrixes, and then complete the projective transforms of $2 \mathrm{D}-3 \mathrm{D}$. In practice, the camera is usually mounted on the terrestrial LiDAR system, and can get a set of 2D color images by rotating around a fixed axis (see Figure 1) (Barnea and Filin, 2007). 


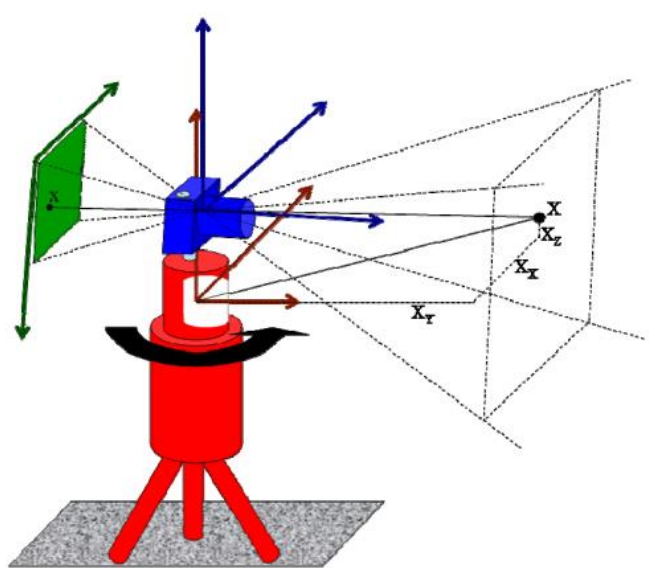

Figure 1. Terrestrial LiDAR system

Due to the rigorous and accurate working model of the mechanism, there are two inherent geometric properties for the terrestrial LiDAR system: one is that all rotation angles between adjacent images are equal, and another is that all relative locations between sequence images coordinates system and LiDAR coordinate system are equal. Therefore, the paper proposed a fast algorithm for calculation of transformation matrix using these properties. The algorithm uses one transformation matrix between one of sequence images and LiDAR point cloud as reference, and then calculates all other transformation matrixes between images and LiDAR point cloud based on these inherent geometric properties. The calculation formula can be expressed use Eq. (3).

$$
\left\{\begin{array}{c}
T_{\text {other }}=T_{\text {reference }} \\
R_{\text {other }}=R_{\text {reference }} R_{\text {angle }}
\end{array}\right.
$$

Where $\boldsymbol{T}_{\text {other }}$ : unknown translation vector between image coordinate system and LiDAR point cloud coordinate system

$\boldsymbol{T}_{\text {reference }}$ : known translation vector (calculated at Section 2.1)

$\boldsymbol{R}_{\text {other }}$ : unknown rotation matrix between image coordinate system and LiDAR point cloud coordinate system (the matrix is expressed using Rodrigues matrix)

$\boldsymbol{R}_{\text {reference }}$ : known rotation matrix between image coordinate system and LiDAR point cloud coordinate system (the matrix is expressed using Rodrigues matrix and calculated at Section 2.1)

$\boldsymbol{R}_{\text {angle }}$ : rotation matrix based on fixed rotation angle (expressed using Rodrigues matrix).

Due to the camera revolves around a fixed axis which is $\mathrm{z}$ axis of LiDAR coordinate system in general, so all images angles related to $\mathrm{x}, \mathrm{y}$ axis of LiDAR coordinate system are invariant. In Eq. (3), the vector related to rotation matrix $\boldsymbol{R}_{\text {angle }}$ can be expressed using $\mathrm{V}=\left[\begin{array}{lll}0 & 0 & \alpha\end{array}\right]$ ( $\alpha$ is the rotation angle between adjacent images).

\section{RESULTS}

The experiment used a terrestrial LiDAR system which includes a camera and laser scanner to get color information and $3 \mathrm{D}$ information in a scene. Before using, the camera needs to be calibrated, and then can get the camera intrinsic parameters $\boldsymbol{R}_{\text {camera }}$ (see Section 2.1). LiDAR scans the scene in a horizontal direction at 0-360 degree (see Figure 2).

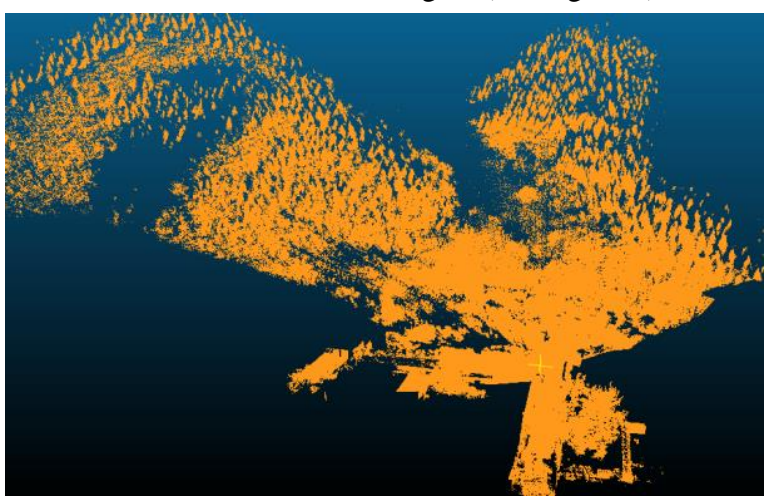

Figure 2. LiDAR point cloud

When laser scanning ended, camera would start taking color images by revolving around the $\mathrm{z}$ axis of LiDAR coordinate system in horizontal direction at 0-360 degree. The rotation angle between adjacent images is 30 degree $\left(\alpha=30^{\circ}\right)$, and can get 12 images (see Figure 3). Therefore, rotation vector of adjacent images can be defined as $\mathrm{V}=\left[\begin{array}{lll}0 & 0 & 30^{\circ} \pi / 180\end{array}\right]$, and can transform in the form of Rodrigues matrix $\boldsymbol{R}_{\text {angle }}$ (see Section 2.2). 

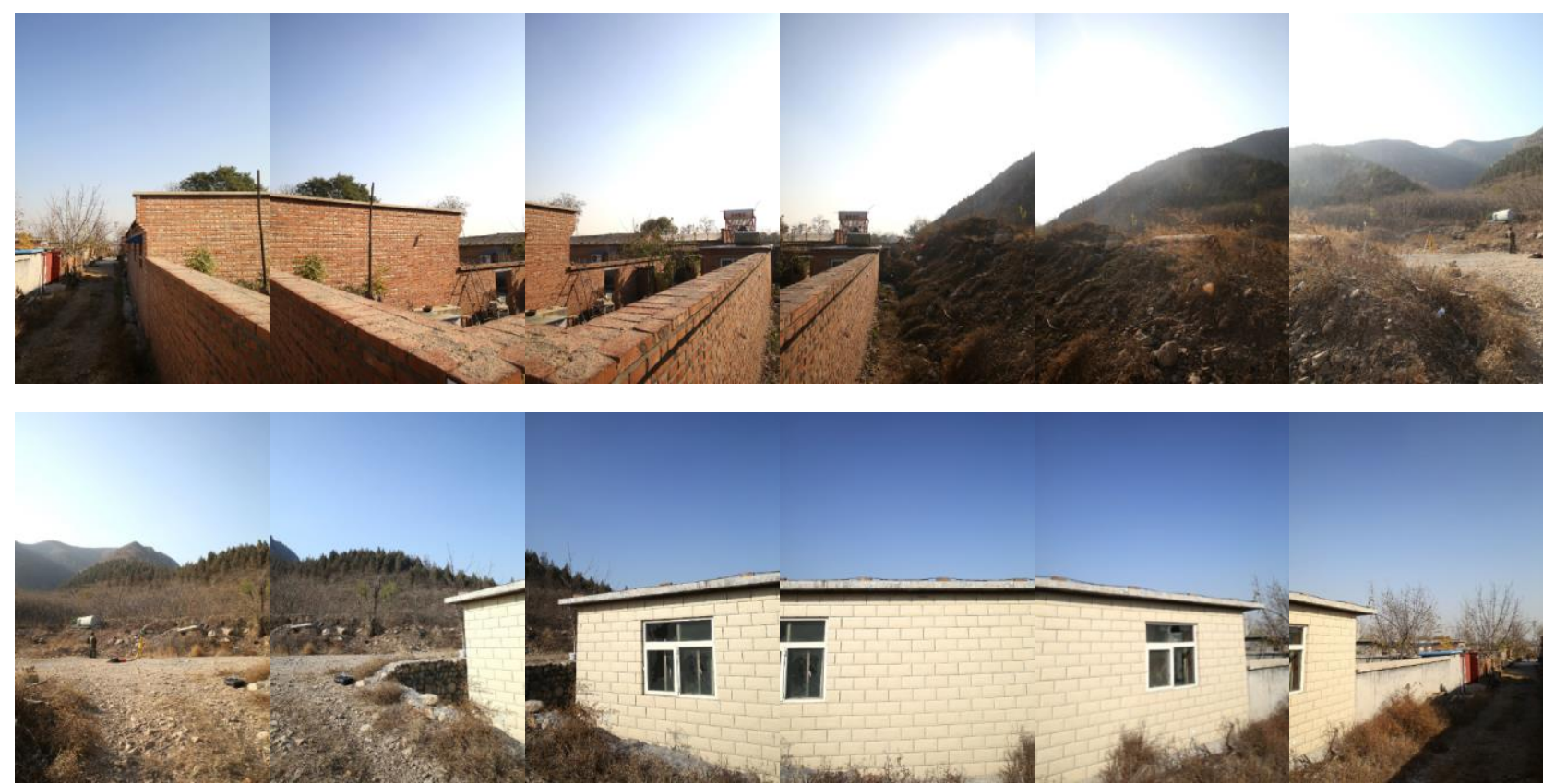

Figure 3. Sequence images

After acquisition of 2D images and 3D LiDAR point cloud using the terrestrial LiDAR system, the experiment selected an image from sequence images randomly, and extracted several pairs of correspondent points from 2D image and LiDAR point cloud by manual. Then, $\boldsymbol{X}_{\text {pixel }}$ and $\boldsymbol{X}_{\text {LiDAR }}$ can be got, and transformation matrix $\boldsymbol{R}_{T}$ can be calculated based on these known parameters (see Section 2.1). When $\boldsymbol{R}_{T}$ was calculated,

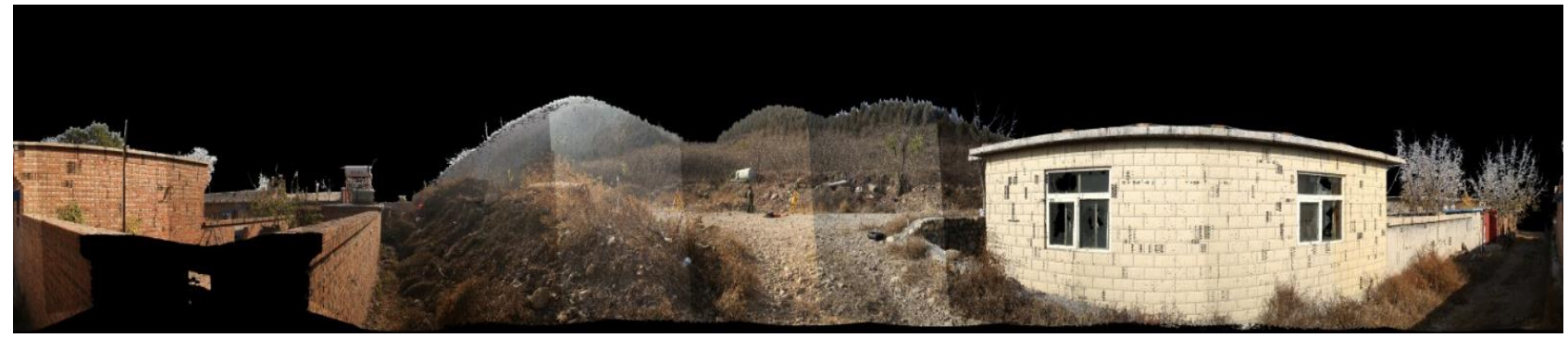

Figure 4. Registration of LiDAR point cloud and sequence images

The Figure 4 shows the registration result which 3D point cloud was projected on a $2 \mathrm{D}$ plane. The registration of LiDAR point cloud and 2D sequence images is accurate, and the overlaps between adjacent images are seamless. It indicated that the method proposed by the paper is reasonable and feasible. we can calculate all other transformation matrixes between images and LiDAR point cloud using Eq. (3). Finally the experiment projected 3D LiDAR point cloud to 2D images by Eq. (1) (see Section 2.1), and completed the registration of LiDAR point cloud and sequence images (see Figure 4). 
transformation matrix between one image and LiDAR point cloud; then, the paper calculated all other transformation matrixes with the fixed rotation angle between sequence images, and completed registration of LiDAR point cloud and sequence images. The registration result is accurate, and overlaps of adjacent images are seamless.

Research basis of the paper is based on a fixed rotation angle of the camera. When the rotation angle is known, the method which proposed by the paper can calculate quickly all transformation matrixes between images and LiDAR point cloud using inherent geometric relation. Meanwhile, the integrated error was decided by the first image and can't occur accumulate error. The method is simple, and the efficiency is high. However, if the rotation angle of the camera is not permanent, the method would be unstable, and appear large error. Therefore, the next research will focus on the unfixed rotation angle.

\section{ACKNOWLEDGEMENTS}

This work was supported by the National Key Research and Development Program of China (NO.2016YFB0501404) and the National Basic Research Program of China (973 Program) Grant No. 2013CB733402. This work was also supported by the National Natural Science Foundation of China Grant Nos. 41331171,41671414 and 41171265.

\section{REFERENCES}

Barnea, S., Filin, S., 2007. Registration of terrestrial laser scans via image based features. International Archives of Photogrammetry, Remote Sensing \& Spatial Information Sciences, pp. 32-37.

Besl, P., Mckay, N., 1992. A method for registration of 3-D shapes. IEEE T. Pattern Anal, 14(2), pp. 239-256.

B“ohm, J., Becker, S., 2007. Automatic marker-free registration of terrestrial laser scans using reflectance features. In: 8th Conference on Optical 3D Measurement Techniques, pp. 338-344.

Li, Y., Low, K., 2009. Automatic registration of color images to 3D geometry. In: Proceeding of the 2009 Computer Graphics International Conference, pp. 21-28.
Liu, L., Stamos, I., Yu, G., Wolberg, G. and Zokai, S., 2006. Multiview geometry for texture mapping 2D images onto 3D range data. In Proceedings of the 2006 IEEE Computer Society Conference on Computer Vision and Pattern Recognition 1, pp. 2293-2300.

Lowe, D., 2004. Distinctive image features from scale-invariant keypoints. Int. J. Comput. Vision, 60(2), pp. 91-110.

Shao, J., Zhang, A.W., Wang, S.M., et al., 2013. Research on fusion of 3D laser point clouds and $\mathrm{CCD}$ image. Chinese Journal of Lasers, 40(5), pp. 228-235.

Sirmacek, B., Lindenbergh, Roderik., Menenti, M., 2013. Automatic registration of iphone images to laser point clouds of urban structures using shape features. ISPRS Annals of the Photogrammetry, Remote Sensing and Spatial Information Sciences, Volume II-5.W2, pp. 265-270.

Swat, A., Veltkamp, R.C., Tan, R.T., 2011. Refined non-rigid registration of a panoramic image sequence to a LiDAR point cloud. Photogrammetric Image Analysisi-ISPRS Conference, pp. 73-84.

Mishra, R., Zhang, Y., 2012. A review of optical imagery and airborne LiDAR data registration methods. Open Remote Sensing Journal, 5(1), pp. 54-63.

Zhang, W.M., Zhao, J., Chen, M., et al., 2015. Registration of optical imagery and LiDAR data using an inherent geometrical constraint. Optical Express, 23(6), pp. 7694-7702.

Zhao, W., Nister, D., Hsu, S., 2005. Alignment of continuous video onto 3D point clouds. IEEE T. Pattern Anal, 27(8), pp. 1305-1318. 